$\Rightarrow$ TECHNIQUE

\section{A mosaic of enhancer function in single cells}

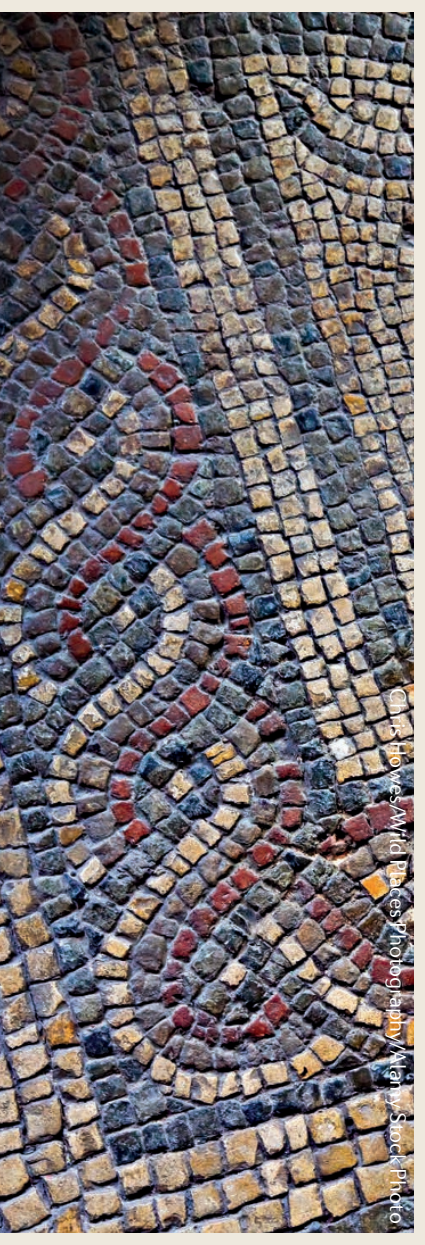

With more than 2 million predicted enhancers interacting in unique genetic and epigenetic contexts, genome-wide functional assessment of these regulatory elements has lagged behind identification. To overcome this issue, a study in Molecular Cell reports the development of Mosaic-seq - short for mosaic single-cell analysis by indexed CRISPR sequencing - which analyses at the single-cell level changes in the transcriptome mediated via targeted enhancer repression.

The strategy uses a catalytically inactive Cas 9 ( $d$ Cas 9 ) fused to KRAB, a strong repressor of enhancer function, and a barcoded single guide RNA (sgRNA) library to generate a mosaic of cells with targeted epigenetic alterations resulting in differing gene expression. Phenotypic characterization was carried out by single-cell RNA sequencing (RNA-seq), and the single-cell transcriptome profiles were correlated with the enhancers targeted by the barcoded sgRNAs.

To validate their approach, the group targeted the $\beta$-globin locus in $\mathrm{K} 562$ cells. Recruitment of dCas9-KRAB to the enhancer HS2 led to repression of gene expression of its downstream targets HBG1, HBG2 and HBE1 to a similar degree to that achieved if the promoters of these genes were targeted directly.
To ensure high quality at large scales, the team generated gold-standard data sets using a TNF $\alpha$ stimulation system in $K 562$ cells using both single-cell and bulk-cell RNA-seq approaches. By examining the distribution of gene expression changes, Mosaic-seq could be used for 'virtual fluorescence-activated cell sorting', with the ability to use as few as 300 cells to detect differential expression of low and moderately expressed genes.

After validation, the researchers applied Mosaic-seq to the functional interrogation of 71 constituent enhancers from 15 super-enhancers, asking whether each constituent enhancer contributes equally or whether a subset contributes more to gene regulation. With a focus on $D N a s e$ I-hypersensitive sites in the $\beta$-globin locus control region, the team were able to confirm previous results that the enhancer HS2 is the major contributor to gene expression. Moving to super-enhancers flanking the gene PIM1, the team could identify those DNase I-hypersensitive regions that contribute to most gene expression.

Using the Mosaic-seq data set, the researchers were able to investigate general features of enhancers. Looking at epigenetic features of active enhancers in more than 500 chromatin immunoprecipitation followed by sequencing (ChlP-seq) data sets, the team noted that the histone acetyltransferase $\mathrm{p} 300$ and RNA polymerase II, along with the sequence-specific transcription factors TAL1 and GATA-2, were enriched at KRAB-responsive enhancers. Moreover, the authors built a statistical model (based on the single-cell penetrance and gene expression contribution of an enhancer) that reflects endogenous enhancer usage.

Finally, Xie et al. wondered what role might be played by constituent components of super-enhancers that have little influence on gene expression. To explore this question, combinations of sgRNAs were used to target multiple constituents of super-enhancers simultaneously. The repression of multiple individual components was shown to be significantly greater than that of individual constituents, suggestive of a compensatory relationship between weak enhancers.

Whereas techniques exist that merge single-cell RNA sequencing with genome editing, such as Perturb-seq and CRISP-seq, the analysis of enhancers is hampered by the small number of target genes for each enhancer. Although Xie et al. acknowledge the limitations of their system — such as whether KRAB is a universal repressor - Mosaic-seq enables genome-wide functional analysis of enhancers and super-enhancers with an unbiased, scalable and combinatorial single-cell approach.

Ross Cloney, Senior Editor, Nature Communications

ORIGINAL ARTICLE Xie, S. et al. Multiplexed engineering and analysis of combinatorial enhancer activity in single cells. Mol. Cell http:// dx.doi.org/10.1016/j.molcel.2017.03.007 (2017) FURTHER READING Burgess, D. J. et al. Combining CRISPR perturbations and RNA-seq. Nat. Rev. Genet. 18, 67 (2017) 